\title{
Mesenteric Lymphangioma Presenting With Small Bowel Volvulus in an Adult
}

\author{
Mohammed Barghash ${ }^{1}$, Suad Nassif ${ }^{1}$, Yazan Alkurdi ${ }^{1}$, Moustafa Mansour ${ }^{1}$ \\ 1. General Surgery, North Manchester General Hospital, Manchester, GBR
}

Corresponding author: Mohammed Barghash, mohammed.barghash@nhs.net

\begin{abstract}
Benign tumours of vascular and lymphatic origin are known as lymphangiomas. In this report, we present a case of a 26-year-old lady admitted with symptoms of small bowel obstruction. Her computed tomography (CT) scan showed a well-defined mass in the small bowel mesentery associated with small bowel volvulus. Segmental resection of the bowel, including the mass, was performed. Microscopic examination and immunohistochemistry of the specimen were consistent with lymphangioma of the small bowel mesentery.
\end{abstract}

Categories: Radiology, Gastroenterology, General Surgery

Keywords: mesenteric lymphangioma, small bowel volvulus, lymphangioma, volvulus, bowel obstruction

\section{Introduction}

Lymphangiomas are benign tumours arising from the vascular and lymphatic origin [1]. They are usually multilocular and may contain serous, serosanguinous, or chylous fluid [2]. Although lymphangiomas are frequently located in the neck, when located intraperitoneally, the small bowel mesentery has been found to be the commonest site, accounting for around $70 \%$ of cases [3].

Small bowel volvulus is one of the causes of bowel obstruction as a result of torsion of the small bowel and its mesentery. It is a well-recognised condition that might affect infants and children; however, it appears to be a rare disease in adults [4]. In this report, we present a case of mesenteric lymphangioma which presented with small bowel obstruction due to volvulus in an adult lady.

\section{Case Presentation}

A 26-year-old lady presented to the accident and emergency (A\&E) department with a 24-hour history of left-sided abdominal pain. There was no associated nausea or vomiting. She also reported opening her bowels 24 hours prior to the presentation. She was a smoker (seven to 10 cigarettes per day) but denied alcohol consumption. Upon questioning, she also gave a three-month history of vaginal bleeding following a medically induced abortion.

Review began 07/09/2021 Review ended 07/24/2021 Published 07/31/2021

๑) Copyright 2021

Barghash et al. This is an open access article distributed under the terms of the Creative Commons Attribution License CC-BY 4.0., which permits unrestricted use, distribution, and reproduction in any medium, provided the original author and source are credited.
On clinical examination, she had abdominal distension and tenderness confined to the left iliac fossa and left flank. Her blood tests showed leucocytosis; however, her liver function tests (LFTs), urea and electrolytes (U\&Es), amylase, C-reactive protein (CRP), and lactate were unremarkable.

A computed tomography (CT) scan of the chest, abdomen, and pelvis was arranged and showed a lobulated well-defined low-density mass in the mesentery measuring approximately $7 \mathrm{~cm}$ in diameter and was associated with a number of prominent vessels that extended into the matrix of the lesion. There was also an associated small bowel volvulus with compression of the superior mesenteric vein (SMV) at the level of the mesenteric root (Figures 1-4). 


\section{Cureus}

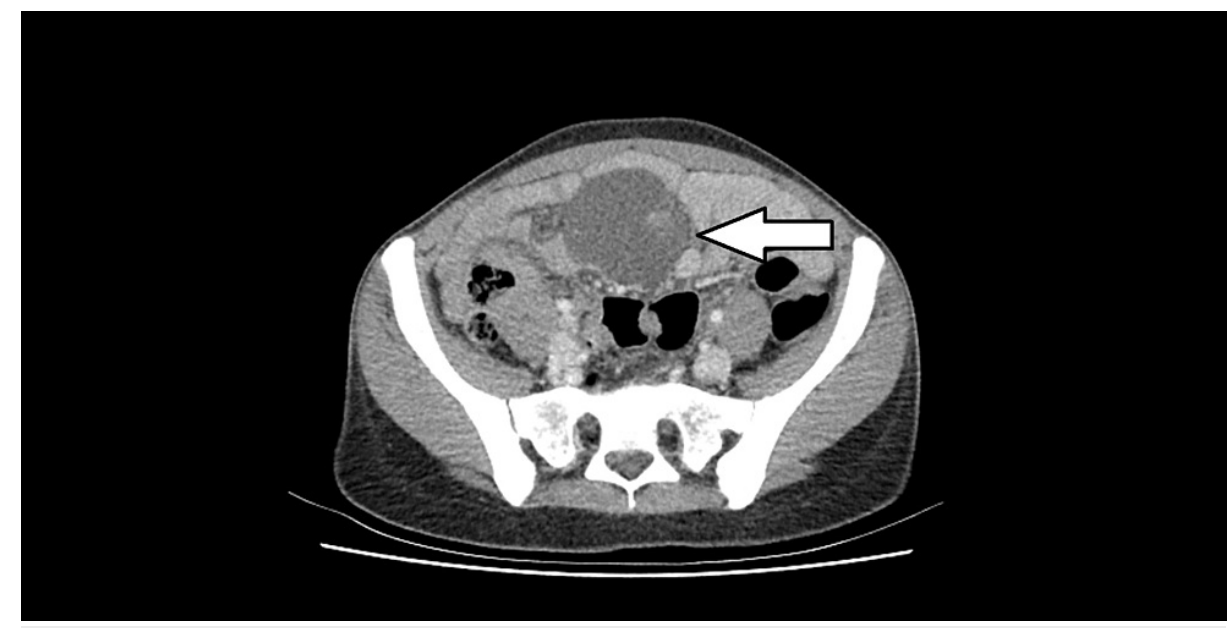

FIGURE 1: An axial abdominal CT scan image showing lobulated, fluidattenuating mass. Mass closely abuts on small bowel loops.

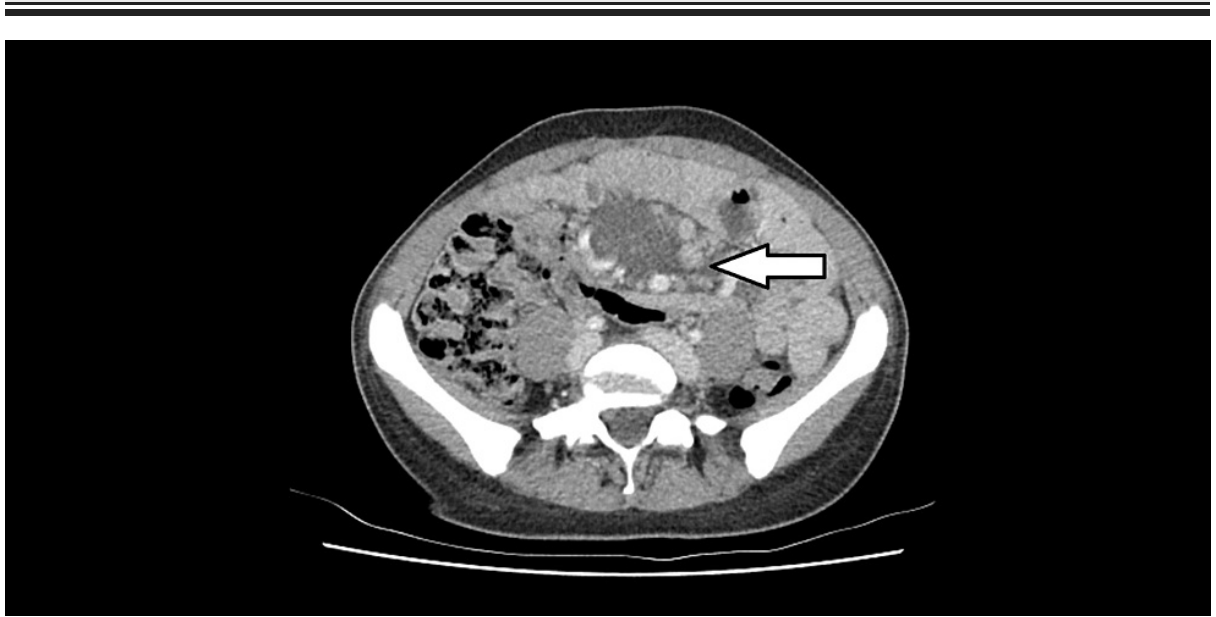

FIGURE 2: An axial abdominal CT scan image showing the mass closely related to a number of prominent vessels

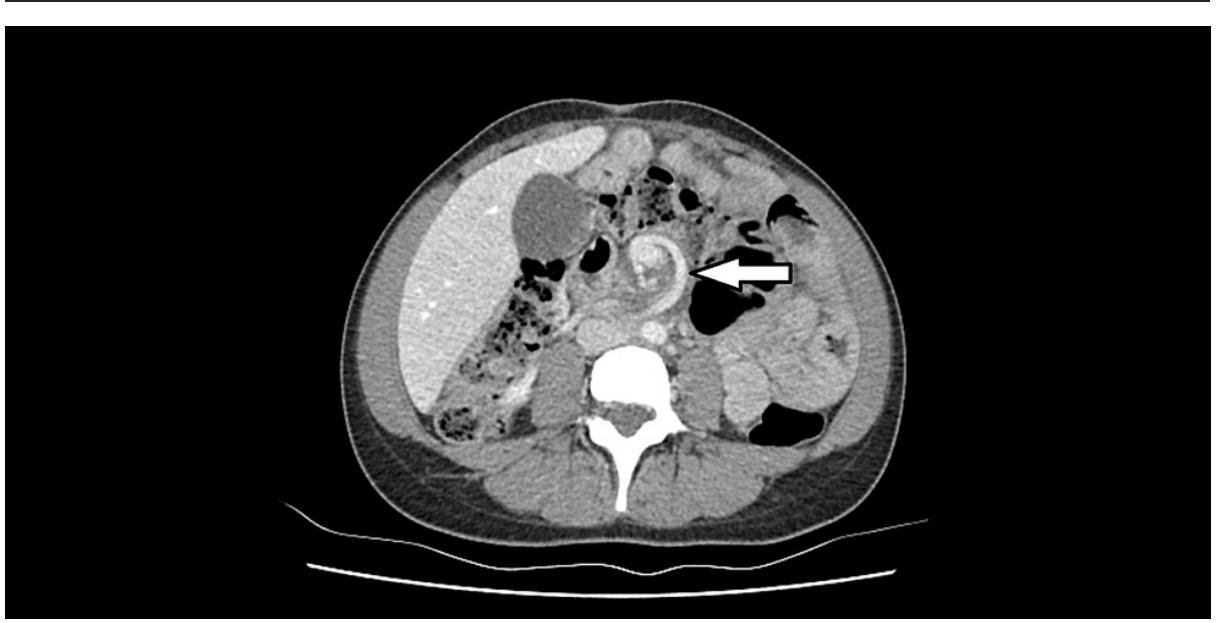

FIGURE 3: An axial CT image showing whirling of the small bowel and mesenteric vessels around superior mesenteric vein leading to its congestion 


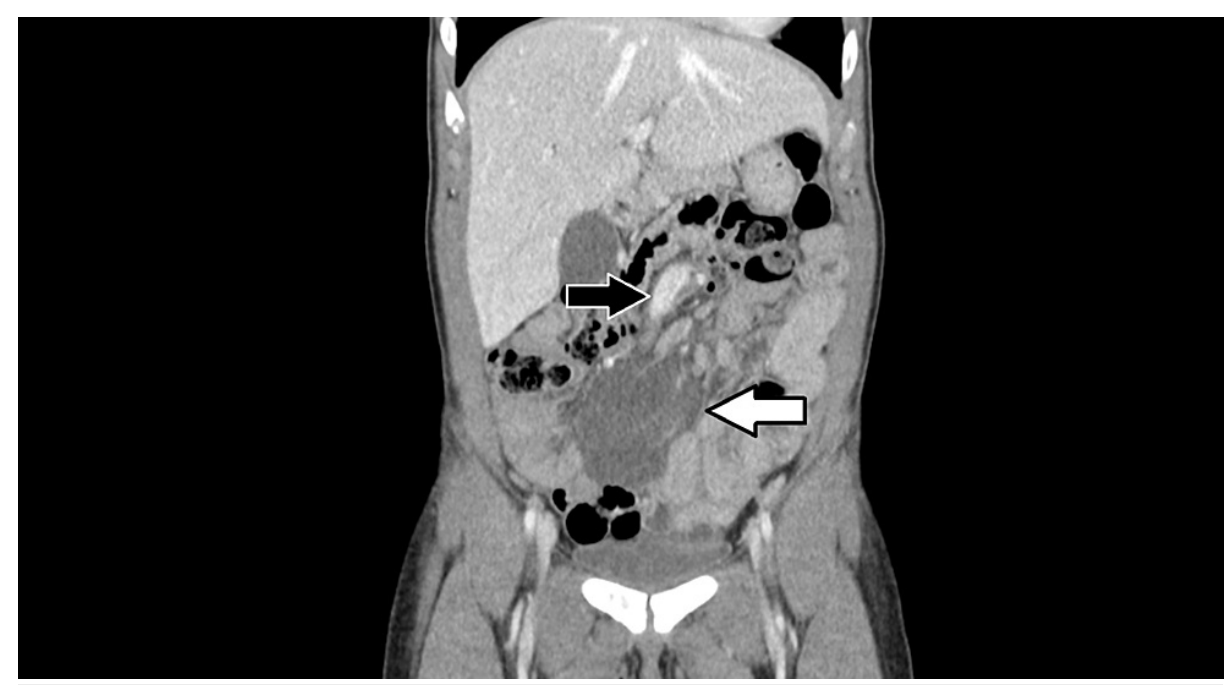

FIGURE 4: A coronal CT image showing a lobulated well-defined lowdensity mass with a number of prominent vessels that extend into the matrix of the lesion (white arrow). Congestion of the superior mesenteric vein (SMV) due to compression can also be observed (black arrow).

After discussion and appropriate consent, the lady was taken to theatre for diagnostic laparoscopy which revealed a small amount of chylous fluid in the pelvis associated with a proximal jejunal mesenteric mass and scattered mesenteric rubbery lymph-nodes. The mass was causing a proximal mesenteric twist. Moreover, it was tethered to the root of the mesentery so laparoscopic mobilisation was unachievable. Therefore, conversion to open surgery was performed uneventfully using a midline laparotomy incision. The twist was undone and the small bowel jejunal segment was excised together with the whole mass and side to side primary anastomosis was performed using a linear stapler.

The postoperative period was uneventful. She improved steadily over a few days. She was able to tolerate oral intake and opened her bowels. Consequently, she was discharged on the fifth postoperative day with a planned follow-up in eight weeks' time.

Microscopic examination of the specimen showed a vasoformative lesion within the small bowel mesentery, composed of dilated, thin-walled channels and cystic spaces lined by cytologically bland, flat endothelial cells and containing proteinaceous fluid along with small lymphocytes and a few red blood cells. Some of the vessel walls contained bundles of smooth muscle. Small lymphocytes were present throughout the septa separating the vessels, with the formation of numerous lymphoid follicles. The lesion expanded the mesentery, extending from just beneath the serosa up to the muscularis propria focally. The separately sampled lymph nodes showed reactive changes. On immunohistochemistry, the endothelial cells were positive for podoplanin (D2-40) and factor VII. Immunostaining for human melanoma black-45 (HMB45) and calretinin was negative. Further stains showed diffuse positivity with a cluster of differentiation-13 (CD31) within the endothelial cells, actin, desmin, calponin, and caldesmon highlight bundles of smooth muscle in the vessel walls. AE1/AE3 cytokeratins and cytokeratin 5/6 (CK5/6) were negative. A diagnosis of lymphangioma of the small bowel mesentery was concluded and a referral to the sarcoma multidisciplinary team (MDT) meeting was made. After the MDT discussion, it was agreed that there was no evidence of sarcomatous changes.

Unfortunately, because of repeated appointment cancellations by the patient, her follow-up was not possible and she was discharged back to her general practitioner (GP).

\section{Discussion}

Cysts of the mesentery are rare. It was first described by Benevieni in 1507, yet it was not until 1884 when Rokitanski described a chylous cyst of the mesentery [5]. Although lymphangiomas are benign, they can proliferate, become aggressive, and invade into adjacent structures [6]. They are usually multilocular lesions derived from vascular and lymphatic origin and may contain serous, serosanguinous, or chylous fluid $[1,2]$.

Approximately $95 \%$ of lymphangiomas are found in the neck and axilla and the other $5 \%$ occur in the mediastinum and abdominal cavity including the mesentery, retroperitoneum, and bones [4]. When located intraperitoneally, around 70\% of cases are located within the small bowel mesentery [3]. Abdominal 
lymphangioma is more common in men than women, and more than $80 \%$ of cases are diagnosed during childhood [3].

The aetiology of lymphangiomas is unknown. Multiple theories have been found including embryonal origin, retention, endothelial secretory disruption, inflammatory, lymph node degeneration, trauma, surgery, and radiation therapy [3,5]. Wegner classified lymphangiomas into simple, cavernous, and cystic [3]. It is worth mentioning that only the cavernous and the cystic types have the potential for malignant transformation [3].

Histologically, lymphangiomas are differentiated from mesenteric cysts by the presence of endothelial lining, connective tissue, and smooth muscle fibres in the former and the cuboidal or columnar lining with a lack of smooth muscles in the latter [2]. Immunohistochemistry includes CD45 factor VIII-related antigen, CD31, CD43, lymphatic vessel endothelial receptor-1, vascular endothelial growth factor-3, prox-1, and monoclonal antibody D2-40, HMB-45, desmin, and calretinin $[7,8]$.

Mesenteric lymphangioma may be discovered incidentally or manifests as intestinal obstruction, volvulus, intussusception, ischemia, mass effect, gastrointestinal bleeding resulting in anaemia, or rupture and shock $[2,3,6,7,9,10]$. Abdominal pain is the most reported symptom [7]. Other symptoms may include abdominal distention, nausea, vomiting, diarrhoea, constipation, or a palpable mass [2]. Differential diagnoses include mesenteric cysts, duplication cysts, ovarian cysts, hydatid cysts, tuberculosis, bowel tumours, and other intraabdominal malignancies $[7,11]$.

The definitive diagnosis of mesenteric lymphangioma is made by histopathological examination, nevertheless, imaging may aid in the diagnosis. Sonographic examination classically shows an anechoic cyst with multiloculated and occasional echogenic debris [1]. Computed tomography (CT) of these masses demonstrates peripheral enhancement, and low or negative attenuation values correlating with the type of fluid content [3]. Magnetic resonance imaging (MRI) is the most accurate imaging modality as it allows the differentiation between mesenteric cysts and lymphangiomas [3].

Small bowel volvulus is a rare condition among adults and is usually classified into two categories [12]. The primary type occurs when there is a segmental twist of the small bowel mesentery without evidence of any predisposing anatomical abnormalities. When it is precipitated by any anatomical abnormality, it is referred to as the secondary type. These precipitating factors include postoperative adhesion, malrotation, congenital bands, intussusception, colostomy, fistula, tumors, and Meckel's diverticulum [12]. Although rare, rotation of a large mesenteric mass can occur and lead to volvulus of the connected mesentery and small bowel resulting in a closed-loop obstruction [4].

There are two theories explaining the relation between small bowel volvulus and lymphangioma. It was argued that the flaccid and mobile nature of a mesenteric lymphangioma can result in its rotation which induces small bowel volvulus. The other theory implies that longstanding or intermittent volvulus causes lymphatic obstruction which results in lymphatic cysts. These cysts are generally unilocular cystic masses without internal septations [4].

While there have been reported cases of spontaneous regression in size of mesenteric lymphangioma, management is mainly surgical aiming at complete surgical resection whenever feasible even in asymptomatic patients, however, some advocate follow up and surveillance in incidentally discovered cases $[3,6,11]$. Surgery can be done by open approach as well as laparoscopically even in children and pregnant women $[3,13]$. In most cases, segmental bowel resection is required [3]. Some patients required pancreaticoduodenectomy as the masses were adherent to the stomach or pancreas [14]. If complete resection is achieved prognosis is excellent, yet recurrence can happen and long-term follow up with an ultrasound scan is advisable $[5,11]$. Sclerotherapy with multiple agents including alcohol and doxycycline had variable results ranging from complete disappearance to recurrence $[3,11]$. Chemotherapy and radiotherapy showed no benefit [15]. Adjuvant therapy with picibanil (OK-432) might halt additional growth in size in unresectable intraabdominal lymphangiomas [16].

\section{Conclusions}

In this study, we presented a case of mesenteric lymphangioma associated with small bowel volvulus in an adult. Complete surgical excision along with the affected small bowel segment was achieved through laparotomy. After the histopathological and immunohistochemical assessment, the diagnosis of mesenteric lymphangioma was established. We recommend follow up with imaging such as abdominal ultrasound, CT, or MRI scans as recurrence might occur.

\section{Additional Information}

\section{Disclosures}

Human subjects: Consent was obtained or waived by all participants in this study. Conflicts of interest: In compliance with the ICMJE uniform disclosure form, all authors declare the following: Payment/services info: All authors have declared that no financial support was received from any organization for the 
submitted work. Financial relationships: All authors have declared that they have no financial relationships at present or within the previous three years with any organizations that might have an interest in the submitted work. Other relationships: All authors have declared that there are no other relationships or activities that could appear to have influenced the submitted work.

\section{References}

1. Levy AD, Cantisani V, Miettinen M: Abdominal lymphangiomas: imaging features with pathologic correlation. AJR Am J Roentgenol. 2004, 182:1485-91. 10.2214/ajr.182.6.1821485

2. Weeda VB, Booij KA, Aronson DC: Mesenteric cystic lymphangioma: a congenital and an acquired anomaly? Two cases and a review of the literature. J Pediatr Surg. 2008, 43:1206-8. 10.1016/j.jpedsurg.2008.01.075

3. Wani I: Mesenteric lymphangioma in adult: a case series with a review of the literature . Dig Dis Sci. 2009, 54:2758-62. 10.1007/s10620-008-0674-3

4. Jang JH, Lee SL, Ku YM, An CH, Chang ED: Small bowel volvulus induced by mesenteric lymphangioma in an adult: a case report. Korean J Radiol. 2009, 10:319-22. 10.3348/kjr.2009.10.3.319

5. Roisman I, Manny J, Fields S, Shiloni E: Intra-abdominal lymphangioma. Br J Surg. 1989, 76:485-9. 10.1002/bjs.1800760519

6. Aliukonis V, Lasinskas M, Pilvelis A, Gradauskas A: Pathological discrepancy: simple mesenteric cyst vs. mesenteric lymphangioma. Case Rep Surg. 2021, 2021:1-5.

7. Abdulraheem AK, Al Sharie AH, Al Shalakhti MH, Alayoub SY, Al-Domaidat HM, El-Qawasmeh AE: Mesenteric cystic lymphangioma: a case report. Int J Surg Case Rep. 2021, 80:105659. 10.1016/j.ijscr.2021.105659

8. Hegazi TM, Al-Sharydah AM, Lee KS, Mortele K: Retroperitoneal cystic masses: magnetic resonance imaging features. Abdom Radiol (NY). 2020, 45:499-511. 10.1007/s00261-019-02246-2

9. Tan B, Zhang SY, Wang YN, Li Y, Shi XH, Qian JM: Jejunal cavernous lymphangioma manifested as gastrointestinal bleeding with hypogammaglobulinemia in adult: a case report and literature review. World J Clin Cases. 2020, 8:140-8. 10.12998/wjcc.v8.i1.140

10. Porras-Ramirez G, Hernandez-Herrera MH: Hemorrhage into mesenteric cyst following trauma as a cause of acute abdomen. J Pediatr Surg. 1991, 26:847-8. 10.1016/0022-3468(91)90153-K

11. Makni A, Chebbi F, Fetirich F, et al.: Surgical management of intra-abdominal cystic lymphangioma. Report of 20 cases. World J Surg. 2012, 36:1037-43. 10.1007/s00268-012-1515-2

12. Huang JC, Shin JS, Huang YT, et al.: Small bowel volvulus among adults. J Gastroenterol Hepatol. 2005, 20:1906-12. 10.1111/j.1440-1746.2005.03945.x

13. Tran NS, Nguyen TL: Laparoscopic management of abdominal lymphatic cyst in children . J Laparoendosc Adv Surg Tech A. 2012, 22:505-7. 10.1089/lap.2012.0003

14. Kitamura H, Yamamoto D, Kadoya S, Bando H, Okayama Y, Minato H: Jejunal mesenteric lymphangioma treated by pancreaticoduodenectomy: a case report. Int J Surg Case Rep. 2020, 77:165-9.

10.1016/j.ijscr.2020.10.088

15. Guivarc'h M: Tumors of the mesentery. Apropos of 102 cases. [Article in French] . Ann Chir. 1994, 48:7-16.

16. Aprea G, Guida F, Canfora A, et al.: Mesenteric cystic lymphangioma in adult: a case series and review of the literature. BMC Surg. 2013, 13:10.1186/1471-2482-13-S1-A4 